EDITORIAL

\title{
LA INGENIERÍA ELECTROMAGNÉTICA MODERNA
}

La ingeniería electromagnética es una rama de la física aplicada, con tal velocidad de desarrollo que en un futuro inmediato los ingenieros electromagnéticos serán indispensables en una nueva e importante área emergente: la ahora denominada Estructura de Onda de la Materia (Wave Structure of Matter, WSM).

La razón principal es la capacidad de penetración de la tecnología electromagnética, donde ingenieros especializados serán necesarios para el diseño de sistemas relacionados con la tecnología WSM. Por ejemplo, en muy altas frecuencias, en sistemas tales como, redes inalámbricas de comunicaciones, chips de computadores, redes ópticas, antenas, y en frecuencias muy bajas, en extracción de energía, en dispositivos de almacenamiento de energía y en sistemas relacionados con un Enfoque Métrico de la Ingeniería (Metric Engineering Approach, MEA), en propulsión con campos electromagnéticos.

La dificultad y la complejidad de las leyes que gobiernan el diseño de sistemas relacionados con la ingeniería electromagnética indican que la teoría y el análisis del electromagnetismo es una ciencia en continua evolución y es un área activa de investigación que ha atraído el interés de matemáticos, científicos de la computación y de los ingenieros. Sin embargo, un buen entendimiento del análisis electromagnético moderno requiere de un profundo conocimiento de la física, habilidad para el análisis matemático y del conocimiento de los algoritmos numéricos utilizados en computación. Aunque algunas universidades enfatizan en el análisis computacional del electromagnetismo, tenemos que ser conscientes de que un estudiante de ingeniería electromagnética debe entender los conceptos de física involucrados y desarrollar intuición y entendimiento de los problemas a resolver. Estas habilidades son importantes tanto para el análisis como para el diseño. Por lo tanto, es importante formar a los estudiantes de postgrado en los métodos modernos del análisis electromagnético, y en las nuevas teorías tales como: metamateriales, electrodinámica quiral y electrogravedad. Por ejemplo, el análisis electromagnético quiral debe incluir, entre otros, los conceptos de ondas polarizadas circularmente, ondas superficiales, ondas que se arrastran (creeping waves), ondas laterales, modos guiados, modos evanescentes, modos radiantes y los modos filtrados (leaky modes). Todo esto en la física de altas frecuencias donde la dualidad onda/partícula emerge como un nuevo enfoque físico de las interacciones electromagnéticas de la WSM.

Recientemente se han producido avances en la WSM, por ejemplo, en microcircuitos industriales y en electrodinámica, donde existen corrientes de lazos cerrados de ondas de electrones, siendo el electrón no una partícula puntual sino una estructura de onda. Aquí la mayoría de las aplicaciones, como ser nanotubos quirales y sustratos de metamateriales para uso en microcircuitos, requiere de la comprensión del comportamiento de la materia en "dimensiones muy pequeñas", donde la aproximación de la partícula falla y la WSM se hace necesaria para entender qué ocurre cuando interactúan diferentes sustratos, a nivel químico, eléctrico o biológico. A nivel de microestructuras, empresas como Intel están empezando a utilizar biología y genética en las técnicas de fabricación de dispositivos orgánicos, usando partes biológicas para sintetizar filamentos quirales de DNA (Deoxyribonucleic acid), donde las ondas que se propagan son equivalentes a las WSM. Por otra parte a nivel macroscópico, para entender adecuadamente la naturaleza de la interacción entre un campo electromagnético de muy alta frecuencia con la materia, debemos considerar la electrodinámica quiral relacionada con la relatividad. Un ejemplo relevante es el diseño de nuevos sistemas GPS (Global Positioning System) con distinta polarización circular, que son más exactos, con la finalidad de mejorar los sistemas actuales.

Un estudiante de electromagnetismo debe estar consciente de la metamorfosis, que ocurre en la física, cuando trabajamos en distintas longitudes de onda o en distintas frecuencias. Cuando la longitud de onda es muy larga, nos encontramos en el dominio de la electro estática y de la magneto estática; aquí se aplica la teoría de circuitos, un ejemplo es el área de los dispositivos de almacenamiento de energía, desde baterías comunes a sofisticados dispositivos híbridos 
utilizados para almacenamiento de energía. Concretamente, en los automóviles modernos, dichos elementos están hechos de mezclas químicas cuyas energías vinculantes son diferentes. Si se conoce la forma en que los elementos de la mezcla se unen, se podrían diseñar baterías para fines específicos, con cálculos basados en la WSM. En el futuro, la WSM requerirá de nuevas técnicas de aplicación, cálculo y diseño de la ingeniería electromagnética. Por otro lado, la mayoría de las aleaciones más valiosas que se utilizan ampliamente en las aplicaciones industriales, como ser el acero, el bronce y el duraluminio, son mezclas simples de elementos básicos, esto es posible gracias a que las uniones de las aleaciones son del tipo Estructuras de Onda.

En relación con todo esto tenemos la MEA, enfoque que será muy importante en las próximas décadas. Esta metodología, para tratar los cambios métricos, ha surgido a través de años de estudio de las teorías electro gravitacionales. Este enfoque es isomórfico con la representación general de la relatividad del vacío, tratando el vacío como un medio polarizable con cambios métricos internos, en términos de la permisividad y la permeabilidad consideradas constantes en el vacío. Este enfoque es básico para obtener energía a partir del vacío (motores magnéticos). Aquí, las ecuaciones de Maxwell en el espacio curvo se modelan como un medio polarizable de índice de refracción variable en el espacio plano, donde la curvatura de un rayo de luz y la reducción de la velocidad de la luz en un potencial gravitacional se representan por un aumento efectivo del índice de refracción. Con este método es posible estudiar los Sistemas de Propulsión de Campos Electromagnéticos, donde la propagación de fotones posee momentum producido por los campos magnéticos y eléctricos ortogonales entre sí (vector de Poynting).

Estos desafíos tecnológicos nos hacen ver que es importante atraer para este campo a personas más calificadas y creativas, reclutando los mejores estudiantes y estimulando su creatividad. En esta perspectiva de la enseñanza de la ingeniería electromagnética, la gente joven siempre puede generar buenas ideas, forjar nuevas fronteras, crear nuevas áreas de trabajo y cultivar el pensamiento independiente, estimulados por el profesor en el desafío de pensar.

Puesto que el análisis electromagnético ha sido usado como una importante herramienta de predicción en muchas ramas de la ingeniería eléctrica, seguirá siendo aún más importante en las nuevas tecnologías. La larga y rica historia del electromagnetismo nos ofrece un desafío sobre cómo debemos educar a nuestros estudiantes de postgrado en esta área. El total del conocimiento requerido no se puede entregar dentro del corto período de su enseñanza universitaria. Por lo tanto, es fundamental educarlos en los conocimientos básicos, ya que aprender todo lo pertinente a la tecnología electromagnética requiere de toda una vida de aprendizaje. Asimismo, es importante educar a dichos estudiantes como pensadores, en vez de adquirir los conocimientos en forma mecánica, siendo esta forma de enseñar un importante aporte para nuestra sociedad.

Es así como, en este número, presentamos el aporte del doctor Héctor Torres-Silva en esta área del electromagnetismo moderno, mediante la electrodinámica vinculada a la mecánica cuántica y a la gravitación. Este trabajo incluye aspectos fundamentales de la WSM, al unificar al electromagnetismo y a la gravitación a través de la electrodinámica quiral, mostrando en este estudio, en forma rigurosa, que la mecánica cuántica de Dirac es una consecuencia lógica de dicha unificación.

\section{Carlos Villarroel González} Editor

Ingeniare. Revista chilena de ingeniería Universidad de Tarapacá Arica, Chile 